\title{
UHPLC-HRMS and GC-MS Screening of a Selection of Synthetic Cannabinoids and Metabolites in Urine of Consumers
}

\author{
Manuela Pellegrini ${ }^{1, \dagger}{ }^{+}$Emilia Marchei ${ }^{1,+}$, Esther Papaseit ${ }^{2,3}$, Magí Farré ${ }^{2,3}{ }^{(0)}$ and \\ Simona Zaami ${ }^{4, *}$ \\ 1 National Centre on Addiction and Doping, Istituto Superiore di Sanità, V.Le Regina Elena 299, 00161 Rome, \\ Italy; manuela.pellegrini@iss.it (M.P.); emilia.marchei@iss.it (E.M.) \\ 2 Clinical Pharmacology Unit, Hospital Universitari Germans Trias i Pujol and Institut de Recerca Germans \\ Trias i Pujol (HUGTiP-IGTP), 08916 Badalona, Spain; epapaseit.germanstrias@gencat.cat (E.P.); \\ mfarre.germanstrias@gencat.cat (M.F.) \\ 3 Department of Pharmacology, Therapeutics and Toxicology, Universitat Autònoma de Barcelona, \\ 08193 Cerdanyola del Vallés, Spain \\ 4 Department of Anatomical, Histological, Forensic and Orthopedic Sciences, Sapienza University, \\ 00161 Rome, Italy \\ * Correspondence: simona.zaami@uniroma1.it; Tel.: +39-0649912226 \\ + Both authors equally contributed to the study.
}

Received: 1 July 2020; Accepted: 12 August 2020; Published: 13 August 2020

\begin{abstract}
Background and Objectives: The use of synthetic cannabinoids has increased around the world. As a result, the implementation of accurate analysis in human biological matrices is relevant and fundamental. Two different analytical technologies, ultra-high-performance liquid chromatography-high-resolution mass spectrometry (UHPLC-HRMS) and high-sensitivity gas chromatography-mass spectrometry (GC-MS) were used for the determination of three synthetic cannabinoids JWH-122, JWH 210, UR-144 and their metabolites in urine of consumers. Materials and Methods: Sample preparation included an initial hydrolysis with $\beta$-glucuronidase and liquid-liquid extraction. The UHPLC-HRMS method included a Kinetex $2.6 \mathrm{u}$ Biphenyl 100A $(100 \times 2.1 \mathrm{~mm}$, $2.6 \mu \mathrm{m})$ (Phenomenex, Italy) column with a gradient mobile phase consisting of mobile phase A (ammonium formate $2 \mathrm{mM}$ in water, $0.1 \%$ formic acid) and mobile phase B (ammonium formate $2 \mathrm{mM}$ in methanol/acetonitrile 50:50 (v/v), 0.1\% formic acid) and a full-scan data-dependent MS2 (ddMS2) mode was used (mass range 100-1000 m/z). The GC-MS method employed an ultra-Inert Intuvo GC column (HP-5MS UI, $30 \mathrm{~m} \times 250 \mu \mathrm{m}$ i.d, film thickness $0.25 \mu \mathrm{m}$; Agilent Technologies, Santa Clara, CA, USA) and electron-impact (EI) mass spectra were recorded in total ion monitoring mode (scan range 40-550 m/z). Results: Both methods have been successfully used for screening of parent synthetic cannabinoids and their metabolites in urine samples of consumers. Conclusions: The screening method applied JWH-122, JWH-210, UR-144 and their metabolites in urine of consumers can be applied to other compounds of the JWH family.
\end{abstract}

Keywords: synthetic cannabinoids; urine; liquid chromatography; high-resolution mass spectrometry; gas chromatography-mass spectrometry

\section{Introduction}

Over the last few years, synthetic cannabinoids, also called synthetic cannabinoid receptor agonists, have been introduced on the illicit market to evade psychotropic drugs legislation and increase the onset and the duration of action of cannabis effects [1,2]. 
The rapid synthesis of such compounds and their rising popularity in illegal markets have become a challenge for clinical and forensic laboratories, since the development of analytical methods cannot keep up with the rapid change of chemical structures. Indeed, 14 chemical families of synthetic cannabinoid receptor agonists are presently recognized and not all are included in current banning laws $[1,3]$. Similar to the other new psychoactive substances, the analytical detection of synthetic cannabinoids presents several limitations, the most important being the unavailability of adequate reference standards for parent drugs and metabolites and the lack of analytical methods to readily detect these substances in cases of intoxication and fatalities [4]. Differently from cannabis products, synthetic cannabinoids can cause severe toxicity in active consumers and the offspring of pregnant or breastfeeding mothers $[5,6]$, causing outbreaks of intoxication and fatalities $[7,8]$. Therefore, the analytical challenge involves not only the large range of different compounds and/or metabolites to identify, but also the variety of biological matrices to investigate including non-conventional matrices, which have gained major interest in recent years for information provided, detection window and minimal sample collection invasiveness [9].

A variety of rapid test kits have been developed and marketed over the past few decades, most of which are only intended for rapid and presumptive identification of traditional drugs of abuse. However, the specificity of these methods depends on the affinity and the cross-reactivity of the antibodies used for the parent drug, its analogues and its metabolites. Initial screening of synthetic cannabinoids and their metabolites in biological matrices is difficult since the few onsite or immunochemical tests available on the market are specific for single molecules and often only for parent drugs [10]. The rapid and continuous release of novel molecules and the need to detect not only parent drugs but also and especially drug metabolites require more specific and selective methodologies such as gas or liquid chromatography coupled to mass spectrometry or tandem mass spectrometry [11-16]. We hereby propose a screening method for urinalysis of synthetic cannabinoids and principal metabolites using a fast sample extraction and two different analytical techniques: ultra-high-performance liquid chromatography-high-resolution mass spectrometry (UHPLC-HRMS) and high-sensitivity gas chromatography-mass spectrometry (GC-MS). The developed methodology has been used for the rapid screening of three synthetic cannabinoids JWH-122, JWH-210 UR-144 and their respective metabolites JWH-122 N-(4-hydroxypentyl), JWH-122 N-(5-hydroxypentyl), JWH-210 N-(4-hydroxypentyl), JWH-210 N-(5-hydroxypentyl), UR-144 N-(4-hydroxypentyl) and UR-144 N-(5-hydroxypentyl) in urine of Spanish consumers. We focused on these particular substances due to their recent spread on the Spanish illegal market, as reported by the same consumers in web fora and to a Spanish Non Governmental Organization dealing with drug risk reduction.

\section{Methodology}

\subsection{Sample Preparation}

Urine samples collected between 29 March 2019 and 10 May 2019 were donated by synthetic cannabinoid consumers, who attended at a private club. Each participant self-administered a cigarette containing the synthetic cannabinoid they selected which was obtained from an unknown source, but analysed for content by a Drug Checking Service performed by a Spanish ONG (Energy Control). At the time of the study, none of these synthetic cannabinoids were illegal in Spain and personal use was allowed in private clubs for cannabis and synthetic cannabinoid users. Sample collection was authorized by the local Human Research Ethics Committee (CEI-HUGTiP ref. PI-18-267, Badalona, Spain). Samples were stored at $-20{ }^{\circ} \mathrm{C}$ until analysis.

Analytes were retrieved by liquid-liquid extraction. Since it has been shown that synthetic cannabinoid hydroxymetabolites are mainly present as glucuronides in urine samples, urine hydrolysis was performed as reported in previous studies $[12,14,15]$. 
A volume of $2 \mathrm{~mL}$ urine was mixed with $200 \mu \mathrm{L} \beta$-glucuronidase (type $\mathrm{H} 2$ from Helix Pomatia $(110,200$ units/mL, Sigma-Aldrich, Milan, Italy) in $0.1 \mathrm{M}$ phosphate buffer (pH 4). Hydrolysis of conjugates was achieved by incubation at $60^{\circ} \mathrm{C}$ for $2 \mathrm{~h}$.

After cooling, samples were extracted twice with $6 \mathrm{~mL}$ hexane/ethyl acetate (9:1). After centrifugation, the organic layer was divided in two aliquots of $3 \mathrm{~mL}$ each.

The first aliquot was evaporated to dryness at $40{ }^{\circ} \mathrm{C}$ under a nitrogen stream and derivatized with $25 \mu \mathrm{L}$ Bis(trimethylsilyl)trifluoroacetamide (BSTFA) containing 1\% trimethylchlorosilane (TMCS) at $70{ }^{\circ} \mathrm{C}$ for $30 \mathrm{~min}$. A volume of $1 \mu \mathrm{L}$ was injected into the GC-MS system.

The second aliquot was evaporated to dryness under a nitrogen stream and then dissolved in $50 \mu \mathrm{L}$ mixture of mobile phase A (ammonium formate $2 \mathrm{mM}$ in water, $0.1 \%$ formic acid) and B (ammonium formate $2 \mathrm{mM}$ in methanol/acetonitrile 50/50, 0.1\% formic acid) (50:50, v/v). A volume of $10 \mu \mathrm{L}$ was injected into UHPLC-HRMS.

\subsection{Gas Chromatography-Mass Spectrometry (GC-MS) Instrumentation}

The GC-MS instrument consisted of an Intuvo 9000 GC System coupled with 5977 B MSD (Agilent Technologies, Palo Alto, CA, USA). The Ultra-Inert Intuvo GC column (HP-5MS UI, $30 \mathrm{~m} \times 250 \mu \mathrm{m}$ i.d, film thickness $0.25 \mu \mathrm{m}$; Agilent Technologies) was used for separation. The GC-MS conditions for the screening procedures were as follows: split-less injection mode; helium (purity 99\%) carrier gas flow $1.2 \mathrm{~mL} / \mathrm{min}$; injection port, ion source, quadrupole, and transfer line temperatures were 260, 230, 150 and $320{ }^{\circ} \mathrm{C}$, respectively; column temperature was $70{ }^{\circ} \mathrm{C}$ for $2 \mathrm{~min}$ and increased to $190^{\circ} \mathrm{C}$ at $30{ }^{\circ} \mathrm{C} / \mathrm{min}$ and then increased to $290{ }^{\circ} \mathrm{C}$ at $5{ }^{\circ} \mathrm{C} / \mathrm{min}$ for $10 \mathrm{~min}$. Subsequently, the temperature was increased to $340{ }^{\circ} \mathrm{C}$ at $40{ }^{\circ} \mathrm{C} / \mathrm{min}$ to eliminate impurities from the column. The electron-impact (EI) mass spectra were recorded in total ion monitoring mode (scan range 40-550 m/z).

Full scan data files were processed by Agilent MassHunter Workstation-Unknowns Analysis (Agilent Technologies). The mass spectra international libraries used for peak identification were NIST Research Library (National Institute of Standards and Technology) and SWGDRUG Library version 3.6 (Scientific Working Group for the Analysis of Seized DrugsWebsiteswgdrug.org).

\subsection{Ultra-High-Performance Liquid Chromatography-High-Resolution Accurate Masses Spectrometry (UHPLC-HRMS) Instrumentation}

The UHPLC/ESI Q-Orbitrap system consisted of an Ultimate 3000 LC pump and an Ultimate 3000 autosampler coupled with a $Q$ Exactive Focus mass spectrometer equipped with a heated electrospray ionization (HESI) probe operating in positive ionization mode, and the system was controlled by Trace finder 4.0 software (ThermoFisher Scientific, Bremen, Germany).

Separation was performed on a Kinetex Biphenyl 100A $(100 \times 2.1 \mathrm{~mm}, 2.6 \mu \mathrm{m})$ (Phenomenex, Italy). The run time was $18 \mathrm{~min}$ with a gradient mobile phase composed of ammonium formate $2 \mathrm{mM}$ in water with $0.1 \%$ formic acid (mobile phase A) and ammonium formate $2 \mathrm{mM}$ in methanol/acetonitrile $50: 50(\mathrm{v} / \mathrm{v})$ with $0.1 \%$ formic acid (mobile phase B) at a flow rate of $0.6 \mathrm{~mL} / \mathrm{min}$. Initial conditions were $20 \% \mathrm{~B}$, held for $2 \mathrm{~min}$, increased to $81.4 \% \mathrm{~B}$ within $9 \mathrm{~min}$, increased to $100 \% \mathrm{~B}$ within $0.2 \mathrm{~min}$, held for $4.3 \mathrm{~min}$, returned to $20 \% \mathrm{~B}$ within $0.1 \mathrm{~min}$, and then held for $2.4 \mathrm{~min}$. LC flow was directed to waste for the first $4.5 \mathrm{~min}$ and after $13.5 \mathrm{~min}$. Autosampler and column oven temperatures were $4{ }^{\circ} \mathrm{C}$ and $40{ }^{\circ} \mathrm{C}$, respectively.

MS parameters were as follows: ionization voltage was $3.0 \mathrm{kV}$; sheath gas and auxiliary gas were 35 and 15 arbitrary units, respectively; S-lens radio frequency RF level was 60; vaporizer temperature and capillary temperature were $320^{\circ} \mathrm{C}$. Nitrogen was used for spray stabilization, for collision induced dissociation experiments in the higher-energy collisional dissociation (HCD) cell and as the damping gas in the C-trap. The instrument was calibrated in positive and negative modes every week.

Data were acquired in full-scan data-dependent MS2 (ddMS2) mode with an inclusion list containing the exact masses of over 1400 compounds including parent compounds and their metabolites. 
Full scan data acquisition was conducted as follows: resolution of 70,000, micro-scans of 1 , maximum injection time of $120 \mathrm{~ms}$ and a scan range of $100-1000 \mathrm{~m} / \mathrm{z}$.

The following settings for the dd-MS ${ }^{2}$ mode were used: resolution of 17,500 , isolation window of 1.0 and HCD cell with stepped normalized collision energy of 17.5, 35.0, $52.5 \mathrm{~V}$.

The MS and fragmentation data acquired were processed by Thermo Scientific TraceFinder software. This specific software performs a thorough interrogation of the database by making use of the built-in database and mass spectral library of over 1400 compounds, retention times, isotope pattern matching, and elemental composition determinations to identify drugs and metabolites.

\subsection{Short Methods Validation}

Although a complete methods validation was not carried out, since proposed methodologies were only intended for initial screening of urine samples, relevant validation parameters were determined following the most recent criteria for method development and validation in analytical toxicology $[17,18]$. Limits of Detection (LODs) and Limits of Quantification (LOQs) were estimated by analyzing a pool of blank urine samples with decreasing concentrations of the spiked analytes and thereafter calculating the signal to noise ratio. LOD was defined as the lowest concentration with good chromatography that yielded a signal-to-noise ratio higher than 3 and LOQ the lowest concentration with a signal-to-noise ratio higher than 10. Limits of detection and quantification, carry over, and selectivity were also calculated, as they are essential parameters for a screening methodology.

\section{Results}

A simple and selective screening analysis with simultaneous use of high-sensitivity GC-MS and UHPLC-HRMS was applied for the determination of synthetic cannabinoids JWH-122, JWH-210 UR-144 and their respective metabolites JWH-122 N-(4-hydroxypentyl), JWH-122 N-(5-hydroxypentyl), JWH-210 N-(4-hydroxypentyl), JWH-210 N-(5-hydroxypentyl), UR-144 N-(4-hydroxypentyl) and UR-144 N-(5-hydroxypentyl) in urine. Particularly, hydroxy metabolites have been chosen as the main metabolites of parent synthetic cannabinoids as reported in previous studies [13-16]. The procedure consisted of a two-hour hydrolysis step followed by an extraction lasting $15 \mathrm{~min}$. Due to the unavailability of glucuronated metabolites, hydrolysis efficiency could not be established, but in agreement with previous studies, after hydrolysis hydroxymetabolite urinary concentration increased by $30 \%$ [14-16].

After sample pretreatment, the first aliquot of extracted samples is immediately injected into the UHPLC-HRMS system, for a 20-min run. The second aliquot undergoes a 30-min derivatization followed by a 20-min GC/MS run. Extracted ion chromatograms from the extraction of 1-mL drug-free urine spiked with $100 \mathrm{ng}$ UR-144, JWH-122, JWH-210 and their metabolites, and three positive urine samples screened with the two different instruments, are shown in Figures 1 and 2.

Retention times monitored $\mathrm{m} / \mathrm{z}$ ions, and limits of detection (LOD) and quantification (LOQ) of the analytes under investigations applying the two different methodologies are reported in Table 1.

Although a proper method validation was not carried out, essential parameters were evaluated. LOD and LOQ obtained for all the analytes under investigation fitted the purpose of the study. No additional peaks due to endogenous substances and carryover interfering with analytes were detected. 

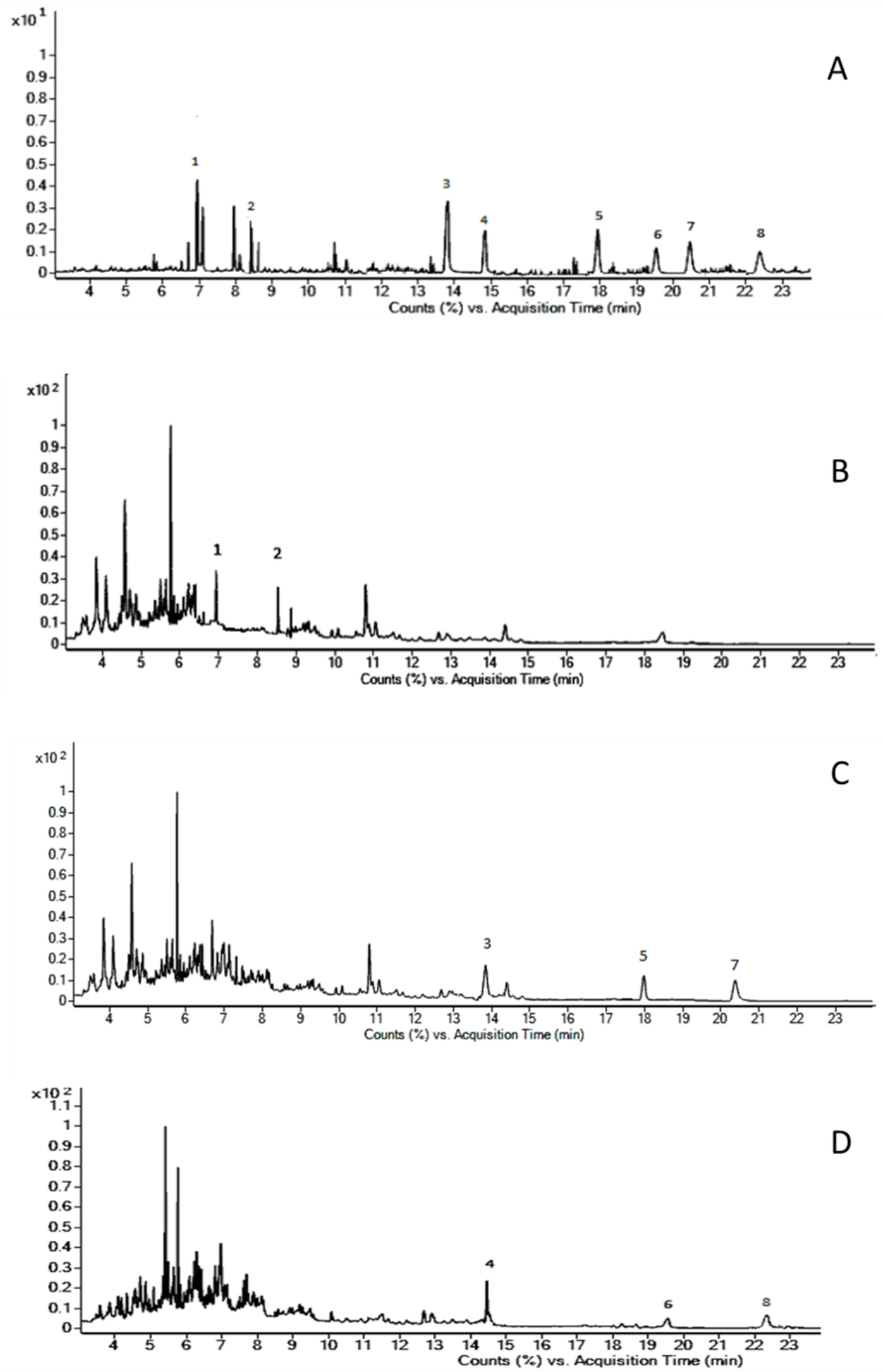

Figure 1. Gas chromatography-mass spectrometry (GC-MS) total ion chromatograms of: (A) Extract of $1 \mathrm{~mL}$ drug-free urine spiked with $0.3 \mathrm{ng}$ of UR-144 (1), UR-144 N-(5-hydroxypentyl) (2), JWH-122 (3), JWH-210 (4), JWH-122 N-(4-hydroxypentyl) (5), JWH-210 N-(4-hydroxypentyl) (6), JWH-122 $\mathrm{N}$-(5-hydroxypentyl) (7) and JWH-210 N-(5-hydroxypentyl) (8). (B) Urine sample positive to UR-144 (1) and UR-144 N-(5-hydroxypentyl) (2); (C) Urine sample positive to JWH-122 (3), JWH-122 $\mathrm{N}-(4$-hydroxypentyl) (5) and JWH-122 N-(5-hydroxypentyl) (7); (D) Urine sample positive to JWH-210 (4) and JWH-210 N-(4-hydroxypentyl) (6) and JWH-210 N-(5-hydroxypentyl) (8). 
5
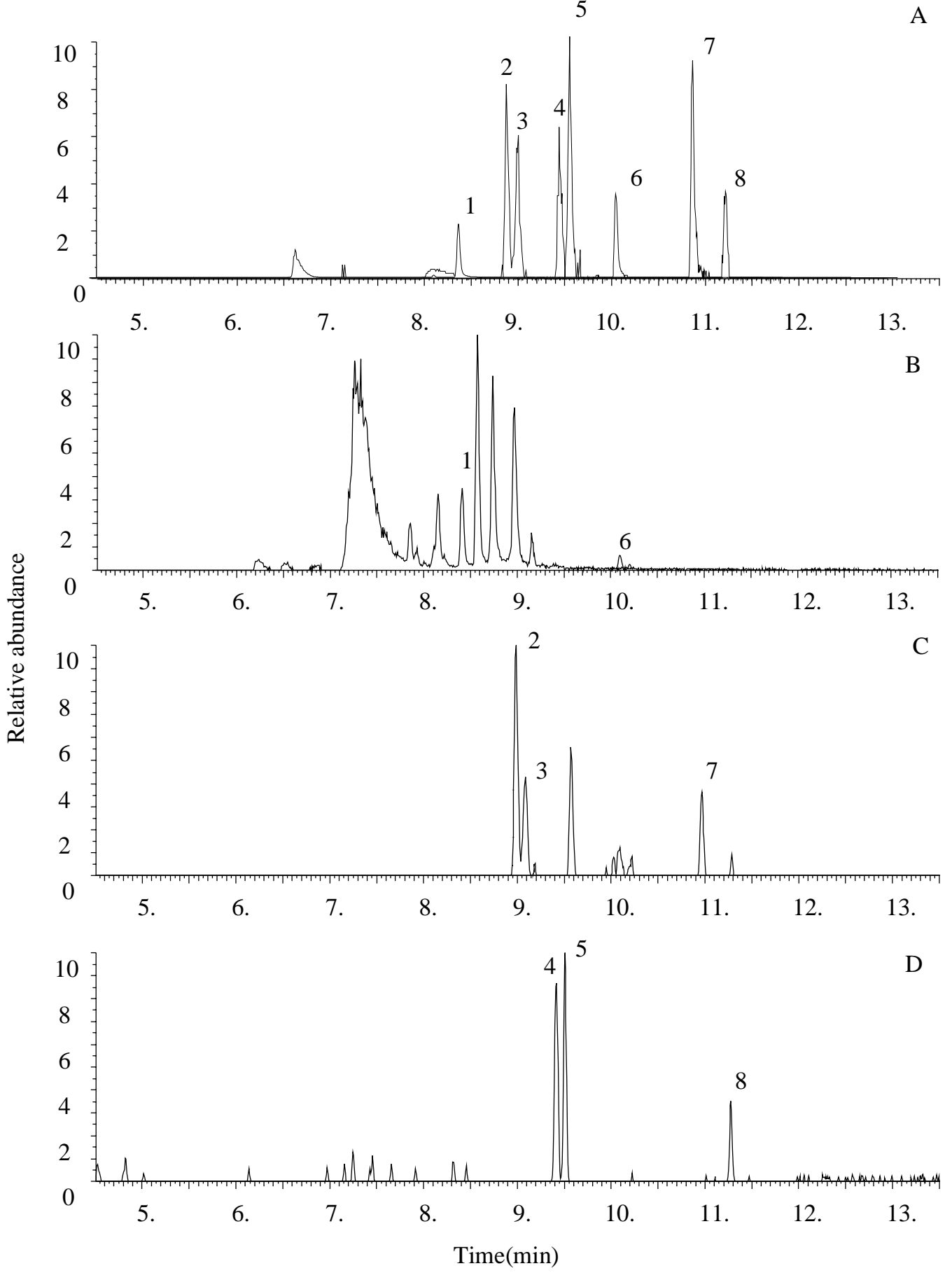

Figure 2. Representative range mass overlay ultra-high-performance liquid chromatography-highresolution mass spectrometry (UHPLC-HRMS) chromatograms of urine. (A) Extract of $1 \mathrm{~mL}$ urine spiked with $0.3 \mathrm{ng}$ of UR-144 5N hydroxy pentyl (1), JWH-122 4N hydroxy pentyl (2), JWH-122 5N hydroxy pentyl (3), JWH-210 4N hydroxy pentyl (4), JWH-210 5N hydroxy pentyl (5), UR-144 (6), JWH-122 (7) and JWH-210 (8), (B) urine sample positive to UR-144 (6) and UR-144 5N hydroxy pentyl (1); (C) Urine sample positive to JWH 122 (7) and JWH-122 4N hydroxy pentyl (2), JWH-122 5N hydroxy pentyl (3); (D) urine sample positive to JWH 210 (8) and JWH-210 4N hydroxy pentyl (4), JWH-210 5N hydroxy pentyl (5). 
Table 1. Retention time (Rt), monitored ions (m/z), Limit of Detection (LOD), Limit of Quantification (LOQ) of non-derivatized and derivatized (TMS) analytes in GC/MS and UHPLC-HRMS.

\begin{tabular}{|c|c|c|c|c|c|c|c|c|}
\hline \multirow[t]{2}{*}{ Compound } & \multicolumn{4}{|c|}{ GC/MS } & \multicolumn{4}{|c|}{ UHPLC-HRMS } \\
\hline & Rt (min) & $\begin{array}{c}\text { Monitored } \\
\text { m/z Ions }\end{array}$ & $\begin{array}{l}\text { LOD } \\
\mathrm{ng} / \mathrm{mL}\end{array}$ & $\begin{array}{c}\mathrm{LOQ} \\
\mathrm{ng} / \mathrm{mL}\end{array}$ & $\begin{array}{c}\mathbf{R t} \\
(\min )\end{array}$ & $\begin{array}{l}\text { Monitored } \\
\mathrm{m} / \mathrm{z} \text { Ions }\end{array}$ & $\begin{array}{l}\text { LOD } \\
\mathrm{ng} / \mathrm{mL}\end{array}$ & $\begin{array}{c}\mathrm{LOQ} \\
\mathrm{ng} / \mathrm{mL}\end{array}$ \\
\hline UR-144 & 7.1 & $144,214,296$ & 0.1 & 0.3 & 10.1 & $\begin{array}{c}55.0551 \\
125.0961 \\
244.0963 \\
312.2321 \\
\end{array}$ & 0.1 & 0.3 \\
\hline $\begin{array}{c}\text { UR-144 } \\
\text { N-(5-hydroxypentyl) } \\
\text { TMS }\end{array}$ & 8.7 & $302,384,399$ & 0.1 & 0.3 & 8.2 & $\begin{array}{l}55.0551 \\
125.0961 \\
230.1172 \\
328.2271\end{array}$ & 0.1 & 0.3 \\
\hline JWH-122 & 13.9 & $214,284,298$ & 0.1 & 0.3 & 10.9 & $\begin{array}{l}144.0443 \\
169.0646 \\
214.1223 \\
356.2008\end{array}$ & 0.1 & 0.3 \\
\hline JWH-210 & 14.8 & $144,183,214$ & 0.1 & 0.3 & 11.2 & $\begin{array}{l}155.0853 \\
183.0803 \\
214.1222 \\
370.2165\end{array}$ & 0.1 & 0.3 \\
\hline $\begin{array}{c}\text { JWH-122 } \\
\text { N-(4-hydroxypentyl) } \\
\text { TMS }\end{array}$ & 18.1 & $169,284,443$ & 0.1 & 0.3 & 9.0 & $\begin{array}{l}141.0697 \\
169.0646 \\
372.1958\end{array}$ & 0.1 & 0.3 \\
\hline $\begin{array}{c}\text { JWH-210 } \\
\text { N-(4-hydroxypentyl) } \\
\text { TMS }\end{array}$ & 19.6 & $183,298,457$ & 0.1 & 0.3 & 9.4 & $\begin{array}{l}144.044 \\
155.0853 \\
183.0803 \\
386.2114\end{array}$ & 0.1 & 0.3 \\
\hline $\begin{array}{c}\text { JWH-122 } \\
\text { N-(5-hydroxypentyl) } \\
\text { TMS }\end{array}$ & 20.6 & $169,284,443$ & 0.1 & 0.3 & 9.0 & $\begin{array}{l}141.0697 \\
169.0646 \\
372.1958\end{array}$ & 0.1 & 0.3 \\
\hline $\begin{array}{c}\text { JWH-210 } \\
\text { N-(5-hydroxypentyl) } \\
\text { TMS }\end{array}$ & 22.6 & $183,298,457$ & 0.1 & 0.3 & 9.5 & $\begin{array}{l}155.0853 \\
183.0803 \\
230.1171 \\
386.2114\end{array}$ & 0.1 & 0.3 \\
\hline
\end{tabular}

Furthermore, even if the total analysis time was not short, this methodology was not set up only to screen the compounds reported in this study, which were only an example. Indeed, preliminary experiments showed that the same methodology could be applied for all JWH families (e.g., JWH 018, JWH 073, JWH 200, JWH 250) tested as pure standards at the time of publication. In this concern it has to be said that two different analytical methodologies with different features were applied to screen the reported compounds. On one hand, UHPLC-HRMS provided a faster run time and it can be applied also in cases where pure standards of parent substances and metabolites under investigation are not available, since molecular ion exact mass measurement and an extended spectra library allowed a good recognition of several synthetic cannabinoids and principal metabolites. On the other, a last generation GC-MS assay provided same sensitivity (in terms of LOD and LOQ) and specificity of UHPLC-HRMS, showing that a more traditional, cheaper, simpler and widespread methodology can also be applied to screen these new psychoactive substances and metabolites in biological fluid.

This evidence is important in clinical and forensic cases involving intoxication or fatalities especially when the consumer is unaware of consumed substances due to surreptitious product substitution or adulteration [19-22].

\section{Conclusions}

The limited availability of screening tests for the detection of synthetic cannabinoids and/or metabolites in urine of consumers $[9,14]$ prompted us to propose a screening method for urinalysis of 
JWH-122, JWH-210, UR-144 and their metabolites. The method can be applied to other compounds of the JWH family and successfully coupled UHPLC-HRMS and GC/MS assays.

Author Contributions: Conceptualization, M.P., E.M. and S.Z.; methodology, E.P., M.F. and E.M.; data curation, all authors; writing —original draft preparation, S.Z. and S.P.; writing—review and editing, all authors. All authors have read and agreed to the published version of the manuscript.

Funding: The investigation was partially funded by grants from the Instituto de Salud Carlos III (ISCIII, Fondo Investigación sanitaria (FIS)-Fondo Europeo de Desarrollo Regional (FEDER), FIS PI14/00715 and FIS PI17/01962, ISCIII-Red de Trastornos Adictivos RTA RD16/0017/0003, and Ministerio de Sanidad, Política Social e Igualdad (Plan Nacional Sobre Drogas-PNSD, 2015I054).

Acknowledgments: The authors thank Laura Martucci, Simonetta di Carlo, Antonella Bacosi, Lourdes Poyatos and Michele Sciotti for technical support.

Conflicts of Interest: The authors declare no conflict of interest.

\section{References}

1. European Monitoring Centre for Drugs and Drug Addiction. Perspectives on Drugs. Synthetic Cannabinoids in Europe. 2016. Available online: http://www.emcdda.europa.eu/attachements.cfm/att_212361_EN_ EMCDDA_POD_2013_Synthetic\%20cannabinoids.pdf (accessed on 31 July 2020).

2. Martinotti, G.; Santacroce, R.; Papanti, D.; Elgharably, Y.; Prilutskaya, M.; Corazza, O. Synthetic Cannabinoids: Psychopharmacology, Clinical Aspects, Psychotic Onset. CNS Neurol. Disord. Drug Targets 2017, 1616, 567-575. [CrossRef] [PubMed]

3. Mazzarino, M.; de la Torre, X.; Botrè, F. A liquid chromatography-mass spectrometry method based on class characteristic fragmentation pathways to detect the class of indole-derivative synthetic cannabinoids in biological samples. Anal. Chim. Acta 2014, 837, 70-82. [CrossRef] [PubMed]

4. Salomone, A.; Vincenti, M.; Gerace, E. Interpretation of NPS results in real hair samples. Toxicol. Anal. Clin. 2017, 29, 4-10. [CrossRef]

5. Solimini, R.; Busardò, F.P.; Rotolo, M.C.; Ricci, S.; Mastrobattista, L.; Mortali, C.; Graziano, S.; Pellegrini, M.; di Luca, N.M.; Palmi, I. Hepatotoxicity associated to synthetic cannabinoids use. Eur. Rev. Med. Pharmacol. Sci. 2017, 21 (Suppl. 1), 1-6.

6. Carlier, J.; Huestis, M.A.; Zaami, S.; Pichini, S.; Busardò, F.P. Monitoring Perinatal Exposure to Cannabis and Synthetic Cannabinoids. Ther. Drug Monit. 2020, 4242, 194-204. [CrossRef]

7. Barceló, B.; Pichini, S.; López-Corominas, V.; Gomila, I.; Yates, C.; Busardò, F.P.; Pellegrini, M. Acute intoxication caused by synthetic cannabinoids 5F-ADB and MMB-2201: A case series. Forensic Sci. Int. 2017, 273, e10-e14. [CrossRef]

8. Paul, A.B.M.; Simms, L.; Amini, S.; Paul, A.E. Teens and Spice: A Review of Adolescent Fatalities Associated with Synthetic Cannabinoid Use. J. Forensic Sci. 2018, 63, 1321-1324. [CrossRef]

9. Giorgetti, A.; Busardò, F.P.; Tittarelli, R.; Auwärter, V.; Giorgetti, R. Post-Mortem Toxicology: A Systematic Review of Death Cases Involving Synthetic Cannabinoid Receptor Agonists. Front. Psychiatry 2020, 11, 464. [CrossRef]

10. Castaneto, M.S.; Wohlfarth, A.; Desrosiers, N.A.; Hartman, R.L.; Gorelick, D.A.; Huestis, M.A. Synthetic cannabinoids pharmacokinetics and detection methods in biological matrices. Drug Metab. Rev. 2015, 47, 124-174. [CrossRef]

11. Graziano, S.; Anzillotti, L.; Mannocchi, G.; Pichini, S.; Busardò, F.P. Screening methods for rapid determination of new psychoactive substances (NPS) in conventional and non-conventional biological matrices. J. Pharm. Biomed. Anal. 2019, 163, 170-179. [CrossRef]

12. Hutter, M.; Broecker, S.; Kneisel, S.; Auwärter, V. Identification of the major urinary metabolites in man of seven synthetic cannabinoids of the aminoalkylindole type present as adulterants in 'herbal mixtures' using LC-MS/MS techniques. J. Mass Spectrom. 2012, 47, 54-65. [CrossRef] [PubMed]

13. Scheidweiler, K.B.; Huestis, M.A. Simultaneous quantification of 20 synthetic cannabinoids and 21 metabolites, and semi-quantification of 12 alkyl hydroxy metabolites in human urine by liquid chromatography-tandem mass spectrometry. J. Chromatogr. A 2014, 1327, 105-117. [CrossRef] [PubMed] 
14. Sadler Simões, S.; Silva, I.; Castañera Ajenjo, A.; João Dias, M. Validation and application of an UPLC-MS/MS method for the quantification of synthetic cannabinoids in urine samples and analysis of seized materials from the Portuguese market. Forensic Sci. Int. 2014, 243, 117-125. [CrossRef] [PubMed]

15. Scheidweiler, K.B.; Michael, J.; Jarvis, Y.; Marilyn, A. Huestis. Nontargeted SWATH acquisition for identifying 47 synthetic cannabinoid metabolites in human urine by liquid chromatography-high-resolution tandem mass spectrometry. Anal. Bioanal. Chem. 2015, 407, 883-897. [CrossRef] [PubMed]

16. Toennes, S.W.; Geraths, A.; Pogoda, W.; Paulke, A.; Wunder, C.; Theunissen, E.L.; Ramaekers, J.G. Excretion of metabolites of the synthetic cannabinoid JWH-018 in urine after controlled inhalation. J. Pharm. Biomed. Anal. 2018, 150, 162-168. [CrossRef] [PubMed]

17. Gundersen, P.O.M.; Spigset, O.; Josefsson, M. Screening, quantification, and confirmation of synthetic cannabinoid metabolites in urine by UHPLC-QTOF-MS. Drug Test. Anal. 2019, 11, 51-67. [CrossRef] [PubMed]

18. Peters, F.T.; Wissenbach, D.K.; Busardò, F.P.; Marchei, E.; Pichini, S. Method development in forensic toxicology. Curr. Pharm. Des. 2017, 23, 5455-5467. [CrossRef] [PubMed]

19. Wille, S.M.R.; Coucke, W.; De Baere, T.; Peters, F.T. Update of standard practices for new method validation in forensic toxicology. Curr. Pharm. Des. 2017, 23, 5442-5454. [CrossRef]

20. Solimini, R.; Rotolo, M.C.; Pellegrini, M.; Minutillo, A.; Pacifici, R.; Busardò, F.P.; Zaami, S. Adulteration Practices of Psychoactive Illicit Drugs: An Updated Review. Curr. Pharm. Biotechnol. 2017, 18, 524-530. [CrossRef]

21. Kyriakou, C.; Pellegrini, M.; García-Algar, O.; Marinelli, E.; Zaami, S. Recent Trends in Analytical Methods to Determine New Psychoactive Substances in Hair. Curr. Neuropharmacol. 2017, 15, 663-681. [CrossRef]

22. Zaami, S. New psychoactive substances: Concerted efforts and common legislative answers for stemming a growing health hazard. Eur. Rev. Med. Pharm. Sci. 2019, 23, 9681-9690.

(C) 2020 by the authors. Licensee MDPI, Basel, Switzerland. This article is an open access article distributed under the terms and conditions of the Creative Commons Attribution (CC BY) license (http://creativecommons.org/licenses/by/4.0/). 